APS

physics

This is the accepted manuscript made available via CHORUS. The article has been published as:

\title{
Observation of precursorlike behavior of femtosecond pulses in a dye with a strong absorption band
}

Matthew M. Springer, Wenlong Yang, Alexandre A. Kolomenski, Hans A. Schuessler, James Strohaber, George W. Kattawar, and Alexei V. Sokolov

Phys. Rev. A 83, 043817 - Published 15 April 2011

DOI: 10.1103/PhysRevA.83.043817 


\title{
Observation of Precursor-Like Behavior of Femtosecond Pulses in a Dye with Strong Absorption Band
}

\author{
Matthew M. Springer,* Wenlong Yang, Alexandre A. Kolomenski, Hans A. \\ Schuessler, James Strohaber, George W. Kattawar, and Alexei V. Sokolov \\ Texas A\&M University
}

\begin{abstract}
Recent interest in Sommerfeld-Brillouin optical precursors has brought attention to the possibility of optical precursor observation in bulk matter. We investigate the possible formation of optical precursors in an organic dye solution with a sharp absorption band and anomalous dispersion at a wavelength of approximately $800 \mathrm{~nm}$. We explore this regime experimentally with sub-10fs pulses with a central wavelength of approximately $800 \mathrm{~nm}$ from a Ti:sapphire oscillator. The pulses are passed through a thin layer of the dye solution and characterized by interferometric autocorrelation. The obtained autocorrelation traces are compared with simulations, and we observe important dispersion effects on the shape of the propagated pulses, including precursor-like behavior in their time evolution.
\end{abstract}

\section{INTRODUCTION}

In the early days of modern physics, Sommerfeld and Brillouin attempted to develop a comprehensive theory of electromagnetic pulse propagation in dielectric media and explored its consistency with the relativistic requirement that the propagation of an electromagnetic signal could not exceed the light speed $c$ in vacuum.[1] Among their theoretical predictions were optical precursors, i.e., parts of the transmitted pulse which generally precede the main signal and whose characteristics are strongly dependant on the dispersion characteristics of the medium. In recent years, electromagnetic pulses have become available with few-cycle rise times which may allow for the possibility of precursor observation in the optical regime. Recent work on this subject has been done in thin semiconductors [2], in atomic gases [3], with electromagnetically induced transparency [4] and slow light [5]. Observations of optical precursors in weakly dispersive bulk media have proved difficult to observe given the usually small variations of the dispersion as a function of wavelength. Because precursor features of a pulse do not attenuate exponentially at the same rate as the main signal [6], the attenuation of femtosecond pulses with distance was explored experimentally in an effort to make such features observable [7]. The subsequent work clarified that non-exponential signal attenuation can be indicative of spectral-domain Bouguer-Lambert-Beer (BLB) absorption rather than precursors [7,8].

The breakdown of the conventional group velocity dispersion approximation in organic dyes with a sharp absorption resonance has been explored experimentally [9], but to our knowledge no direct observation of optical precursors has been obtained in such a dye solution. In this work we numerically and experimentally explore optical precursor phenomena in a linear, dispersive bulk organic dye solution with a sharp absorption resonance by means of simulation of an ultrashort pulse as it propagates through the material. We then compare the numerically modeled precursor dynamics to the measured autocorrelation of the ultrashort pulse after passing through the dye layer in experiment. Close agreement between simulation and experiments indicates that precursor behavior rather than group velocity dispersion is extant in this sharply absorbing organic dye solution.

\section{DISPERSIVE FIELD PROPAGATION}

Given a propagating electromagnetic pulse of electric field $u(x, t)$, with the boundary condition $\frac{\partial u}{\partial x}(0, t)=0$, we can present the complex spectral amplitude $A(\omega)$ as

$$
A(\omega)=\frac{1}{\sqrt{2 \pi}} \int_{-\infty}^{\infty} d t e^{i \omega t} u(0, t)
$$

In a homogeneous, isotropic, linear medium of complex refractive index $\tilde{n}=\eta(\omega)+i \kappa(\omega)$, the subsequent time evolution of the pulse will be determined by the differing phase velocities and spectral intensity attenuations. We

*Electronic address: msprin1@neo.tamu.edu 
describe the phase shifts and spectral intensity attenuation as $e^{i \tilde{k x}}$ with a complex wave number $\tilde{k}(\omega)=\omega / c[\eta(\omega)+$ $i \kappa(\omega)]$, where $\kappa(\omega)=c \alpha(\omega) / 2 \omega$ with the usual distance absorption coefficient $\alpha(\omega)=-\ln \left[I(\omega) / I_{0}(\omega)\right] / d$ for the attenuation over the total dye propagation distance $d$. Considering only the forward-propagating part of the pulse, we find the inverse Fourier transform

$$
u(x, t)=\frac{1}{\sqrt{2 \pi}} \int_{0}^{\infty} d \omega A(\omega) e^{-\alpha(\omega) x} e^{i\left(\frac{\omega}{c} x \eta(\omega)-\omega t\right)}+c . c .
$$

Under the group velocity dispersion (GVD) approximation with the Taylor expansion of the refractive index $\tilde{n}(\omega)$ to first order, the original field $u(x, t)$ is found to follow its forward propagation while retaining the shape of its envelope. However, the GVD approximation is inadequate in frequency regimes which include phenomena such as strong absorption and anomalous dispersion. Thus, the details of the refractive index dispersion are crucial for correct calculation of the temporal evolution of the pulse. Methods for examining this regime analytically range from simple approximate stationary phase methods[10] to Sommerfeld and Brillouin's more advanced contour integration method[1] and the comprehensive and modern treatment of Oughstun and Sherman [11].

Among the conclusions of this analysis is the discovery that pulses with an initial rise time less than or equal to the characteristic medium response time $\delta^{-1}$ will develop precursor fields whose temporal structure does not necessarily resemble the original field [12]. The characteristics of precursor fields which have attracted experimentalists include propagation speeds which approach c, and diminished or even sub-exponential attenuation over distance (6). Again, it is critical to note that only the precursor part of the pulse displays this behavior, while the total integrated energy of the precursor fields plus the main signal fields will continue to obey the BLB law on a frequency-domain basis.

In most bulk media of physical interest an analytical formula for the complex refractive index is not available. Additionally, the real part of complex index associated with dispersion is more difficult to measure than the imaginary part related to linear absorption. We instead measure the intensity absorption coefficient $\alpha(\omega)$ and calculate the real part of the refractive index $\eta(\omega)=\mathfrak{R}\{\tilde{n}(\omega)\}$ using the Kramers-Kronig principal-value integral relationship

$$
\eta(\omega)-1=\frac{2}{\pi} \mathcal{P} \int_{0}^{\infty} \frac{\Omega \kappa(\Omega)}{\Omega^{2}-\omega^{2}} d \Omega .
$$

The Kramers-Kronig relationship connects the real and imaginary parts of the refractive index for causal dielectrics in the linear regime, which may be explored using an unfocused laser of sufficiently low intensity . If, as in this experiment, low-frequency absorption is unknown in advance, the real part of the refractive index remains uncertain up to the addition of a constant. However, the overall delay corresponding to this constant is neither necessary for pulse shape analysis nor generally available in autocorrelation measurements, therefore it will be disregarded. We also define the characteristic dimensionless optical depth $\tau(\omega)=\alpha(\omega) d$ of the dye solution. The optical depth is directly accessible by measurement and allows us to simulate the propagation of the pulse without requiring a separate measurement of the numerical value of $d$. In this case the Kramers-Kronig relationship becomes

$$
\eta(\omega)-1=\frac{c}{\pi d} \mathcal{P} \int_{0}^{\infty} \frac{\tau(\Omega)}{\Omega^{2}-\omega^{2}} d \Omega
$$

The coefficient $\eta(\omega) x$ for the phase shift in the inverse Fourier transform thus becomes

$$
\eta(\omega) x=x+\varphi \frac{c}{\pi} \mathcal{P} \int_{0}^{\infty} \frac{\tau(\Omega)}{\Omega^{2}-\omega^{2}} d \Omega
$$

where $\varphi=x / d$ is the dimensionless fractional propagation distance. For notational convenience, we further define the total refraction

$$
N(\omega) \equiv \frac{c}{\pi} \mathcal{P} \int_{0}^{\infty} \frac{\tau(\Omega)}{\Omega^{2}-\omega^{2}} d \Omega,
$$

which may be used to calculate the relative phase shift $\frac{\omega}{c} N(\omega) \varphi$ for each frequency component. Computation of numerical values of this expression do not require the numerical value of $d$. 


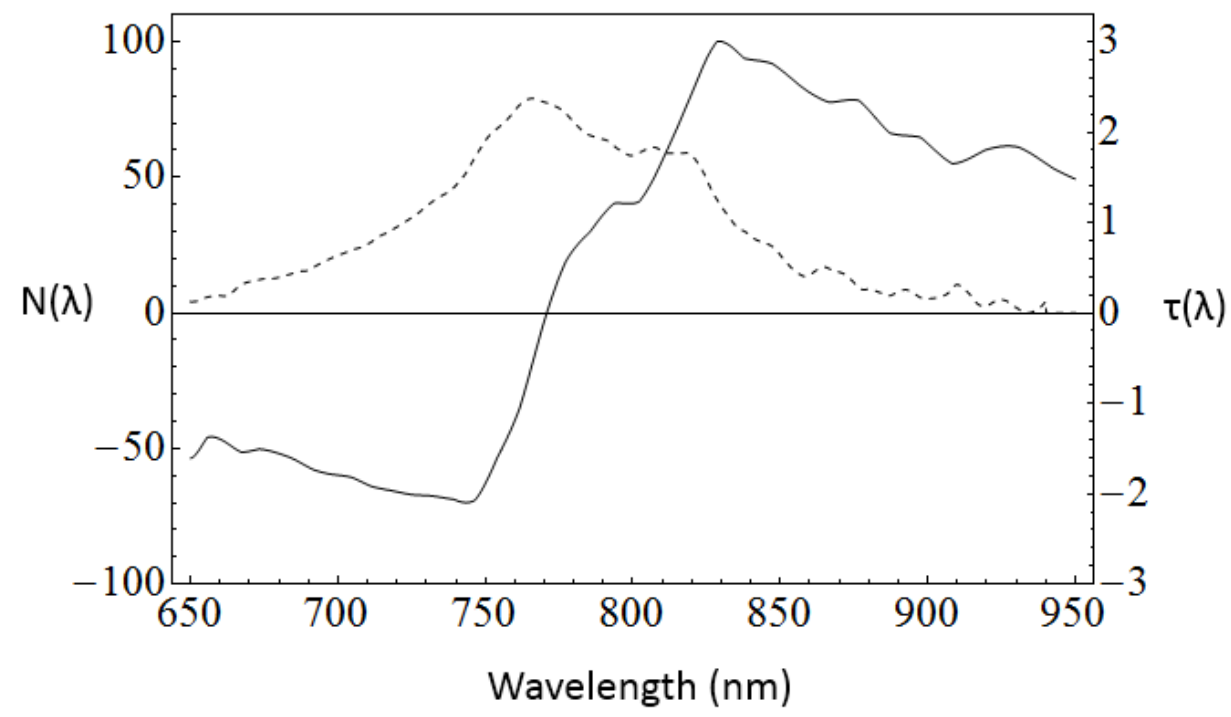

Figure 1: (Color online) Measured absorption curve (dashed line) and calculated total refraction (solid line) of NP800 dye in tetrachloroethane.

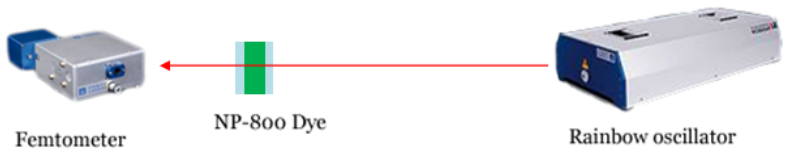

Figure 2: (Color online) Schematic diagram of experiment

\section{EXPERIMENT}

A proprietary dye (NP800, Exciton) selected for its sharp absorption resonance in the near-infrared is used for this experiment. The dye is dissolved in 1-1-2-2-tetrachloroethane for a final dye concentration of $10 \mathrm{~g} / \mathrm{L}$. A thin layer of this solution is sandwiched between two microscope cover slips of approximately 190 micron thickness. The whole sample container is then sealed and inserted into the optical path. The solution is held in place between the cover slips by surface tension. The cover slips, with dye solution suspended in between, are placed onto a support which holds the slips into the path of the beam.

The frequency-dependent intensity absorption coefficient of the dye solution is determined by launching broadspectrum light through the solution and measuring its absorption. Assuming a typical dye solution thickness of 2 $\mu \mathrm{m}$, we then calculate the intensity absorption coefficient, although prior knowledge of the solution thickness is not required for the calculation of the pulse evolution. The absorption curve is shown in Figure 1. We set the absorption to zero for those frequencies with absorption too low to measure.

From this absorption curve we calculate the variation in the real part of the refractive index, with result shown in Figure 1. The Kramers-Kronig relationship ensures that absorption far from the laser bandwidth (such as from the tetrachloroethane solvent) will alter the refractive index within that bandwidth by the addition of a near-constant, and that small errors near and in the absorption band will linearly produce small errors in the calculated real refractive index. Though we plot over a large frequency range for mathematical clarity, it should be noted that only the change in $N(\omega)$ over the bandwidth of the laser is relevant for the simulation.

We then launch the femtosecond laser pulses through the dye solution. The laser pulses are generated by means of a [Rainbow, Femtolasers] femtosecond laser operating with a central wavelength of approximately 800nm, pulse duration of 9 fs temporal FWHM, pulse energy of approximately $2 \mathrm{~nJ}$, and repeition rate of about $80 \mathrm{MHz}$. The low pulse energy ensures that we are exploring the linear regime. We then measure and characterize the pulses using the interferometric autocorrelator [Femtometer, Femtolasers]. By measuring the autocorrelation traces of the initial pulse we are able to characterize its initial temporal shape for later analysis. Finally we measure the autocorrelation trace of the pulse after propagating through the dye solution and cover slips. 


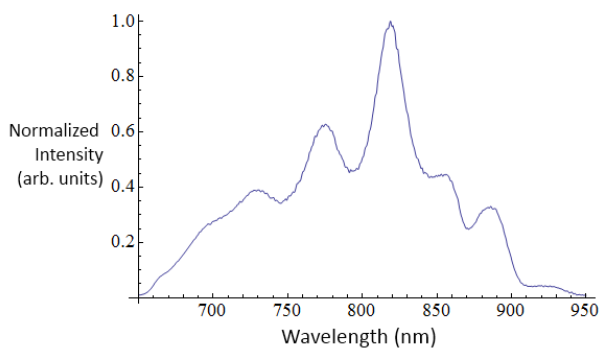

Figure 3: (Color online) Measured intensity spectrum

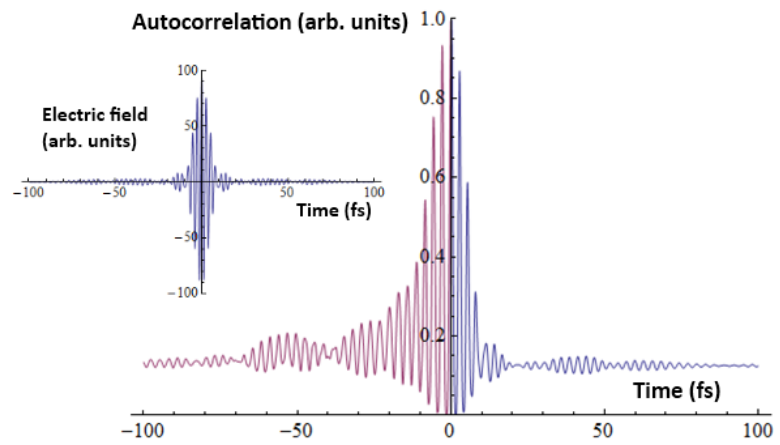

Figure 4: (Color online) Initial electric field guess (inset), with calculated autocorrelation from initial guess (right half of graph, blue) and measured autocorrelation (left half of graph, red).

\section{PULSE CHARACTERIZATION VIA GENETIC ALGORITHM}

In general an analytic method for inverting an interferometric autocorrelation signal to retrieve the original pulse is unknown. Indeed the symmetry of the autocorrelation function implies that there will be at least an ambiguity with respect to time inversion of the pulse, as a generic electric field $\mathrm{E}(\mathrm{t})$ will produce the same autocorrelation as $\mathrm{E}(-\mathrm{t})$ [13]. However, it is nonetheless possible to retrieve certain essential features of the pulse, and in fact the interferometric autocorrelation along with the spectrum is enough to uniquely specify the pulse up to the direction of time ambiguity [14]. The measured spectrum of the pulse is shown in Figure 3. The various deterministic algorithms for pulse shape retrieval tend to stagnate; therefore we attempt to bypass this difficulty by using a nondeterministic genetic algorithm.

Our pulse retrieval method proceeds as follows. We assign a phase function $\phi(\omega)$ defined pointwise over the bandwidth of the pulse, and the phase is varied by the genetic algorithm. From the measured real intensity spectrum $|A(\omega)|^{2}$ we calculate an initial guess for the pulse shape (Figure 4) using the inverse Fourier transform and the assumption that the pulse is transform-limited with a zero phase throughout its spectral bandwidth:

$$
E(t)=\mathfrak{R}\left[\frac{1}{\sqrt{2 \pi}} \int_{-\infty}^{\infty} A(\omega) e^{i \omega t} d \omega\right] .
$$

From this calculated field we determine the interferometric autocorrelation $I(\tau)$ :

$$
I(\tau)=\int_{-\infty}^{\infty}\left|(E(t)+E(t-\tau))^{2}\right|^{2} d t .
$$

The calculated autocorrelation is compared to the measured autocorrelation (Figure 4) and a fitness parameter is determined by the least-squares difference between the calculated and measured autocorrelations. Random perturbation is then applied to the phase function iteratively, until the resultant phase best approximates the observed autocorrelation (Figure 5).

After application of the genetic algorithm we retrieve an approximate spectral phase (Figure 6) and calculate its associated electric field via inverse Fourier transform (Figure 9). 


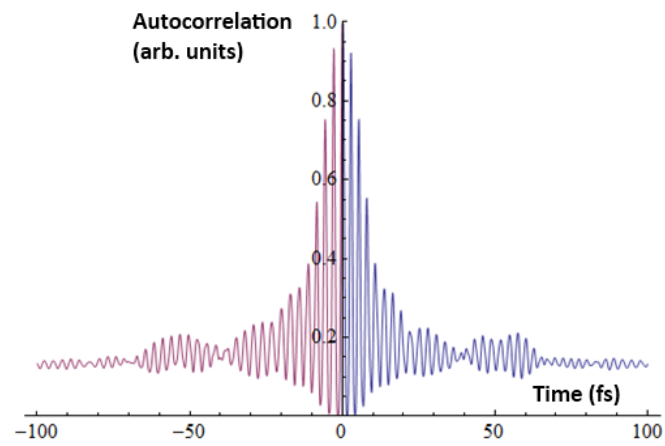

Figure 5: (Color online) Calculated autocorrelation from genetically retrieved pulse (righ half of graph, blue) and measured autocorrelation (left half of graph, red)

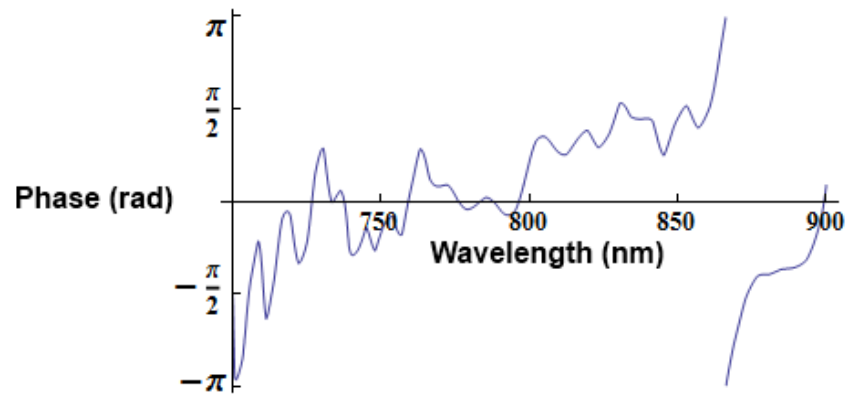

Figure 6: (Color online) Spectral phase retreived from genetic algorithm

The direction of time ambiguity is then eliminated by calculating the shape of the pulse after propagating through the two BK7 glass cover slips in the absence of the dye. The dispersive characteristics of BK7 are well known, and only the correct selection of the direction of time will give the actually observed autocorrelation of the pulse after the glass cover slips. As a secondary check we measure the cross-correlation of the original pulse with the pulse after propagating through the dye. The cross-correlation $I_{x}(\tau)$ is measured by placing the dye solution in one arm of the interferometric autocorrelator and calculated numerically in analogy to the autocorrelation:

$$
I_{x}(\tau)=\int_{-\infty}^{\infty}\left|\left(E_{0}(t)+E_{f}(t-\tau)\right)^{2}\right|^{2} d t
$$

where we distinguish the original field $E_{0}(t)$ and the final field $E_{f}(t)$ after passing through the dye. We compare the measured cross-correlation to the cross-correlation obtained from simulation. The cross-correlation is not as robust a method as autocorrelation due to the fact that two independent sources of error exist - the shape of the original pulse and the shape of the propagated pulse. Nonetheless the technique serves as a convenient secondary check to resolve the direction of time ambiguity.

The resulting retrieved pulse contains significant noise away from the peak. This is an unavoidable consequence of the sensitivity of the inverse Fourier transform to small errors in the retrieved phase. As a consequence we analyze

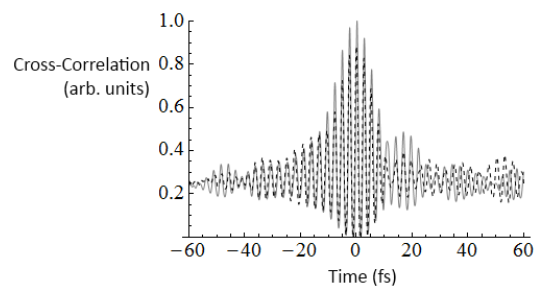

Figure 7: Calculated (dashed curve) and observed (solid gray curve) cross-correlations with correct direction of time 


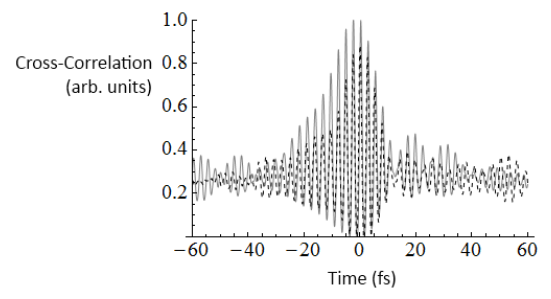

Figure 8: Calculated (dashed curve) and observed (solid gray curve) cross-correlations with incorrect direction of time

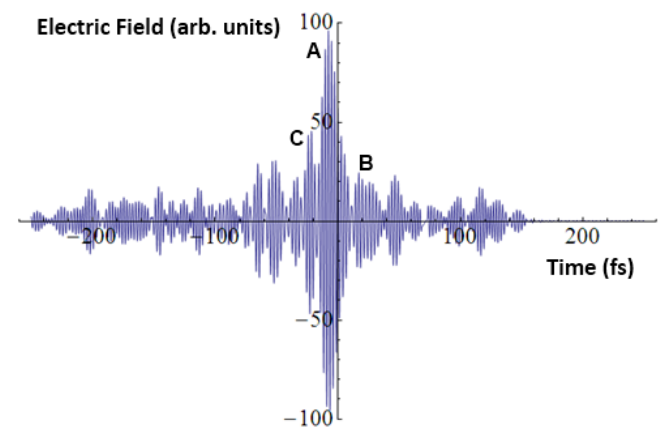

Figure 9: (Color online) Initial E-field determined by genetic algorithm with labeled reference peaks

those pulse features which are large in amplitude and located near the pulse maximum. The 4th power dependence of the interferometric autocorrelation on field strength ensures that these features are the dominant contribution to the autocorrelation and thus less susceptible to noise in the pulse retrieval algorithm. Small features far from the pulse peak should not be assumed to correspond very precisely to the actual laser pulse [15].

\section{ANALYSIS OF SIMULATED PULSE}

The simulation allows us to study the in-situ anatomy of the temporal and spatial behavior, while the experimental measurements validate the simulation. As discussed in the introduction and theory sections, optical precursors can be characterized in terms of their relative velocity to other portions of the pulse and their behavior with regard to distance of propagation. In the latter case the precursor does not necessarily attenuate exponentially with distance, though the pulse as a whole does obey the frequency-domain BLB law. A specific temporal sub-feature of the pulse such as a precursor may "leach" energy from another region of the pulse, maintaining its amplitude at the expense of adjacent parts of the pulse. This should not be taken as a violation of the BLB law, as each spectral component is separately attenuated with distance in the usual way.

Because precursors are closely associated with sharp near-discontinuous features of a pulse, characterization of the attenuation with distance of these features can be indicative of precursors or precursor-like pulse behavior. We therefore select features appearing in the genetically-determined pulse which most closely fit this description and simulate their evolution as a function of distance propagated in the dye. The initial pulse shape determined by the genetic algorithm and shown in Figure 9 contains sharp internal features with rise times on the order of one optical cycle of approximately $2.7 \mathrm{fs}$. The 4 th power dependence of the autocorrelation signal on the field strength leads us to consider the dynamics of the temporally sharp central pulse features of the highest amplitude, labeled in Figure 9.

We simulate the propagation of this pulse using the generalized pulse propagation equation, which we evaluate numerically. Using the definition $\tau(\omega)=\alpha(\omega) d$ and dropping the unknown overall phase $\exp \left[i \frac{\omega}{c} x\right]$ which is not necessary for computing the autocorrelation, we have the propagated pulse

$$
E(t)=\frac{1}{\sqrt{2 \pi}} \int_{0}^{\infty} d \omega A(\omega) e^{-i \omega t} e^{i\left(\frac{\omega}{c} x N(\omega)+i \frac{\tau(\omega)}{2}\right) \varphi} e^{2 n_{g}(\omega) x_{g}}+c . c .
$$

Here $n_{g}(\omega)$ is the real refractive index of the BK7 glass cover slip, and $x_{g}$ is the $190 \mu$ m thickness of one cover slip. We do not include the very small reflection coefficients at the glass/dye interfaces, as the coefficients cannot 


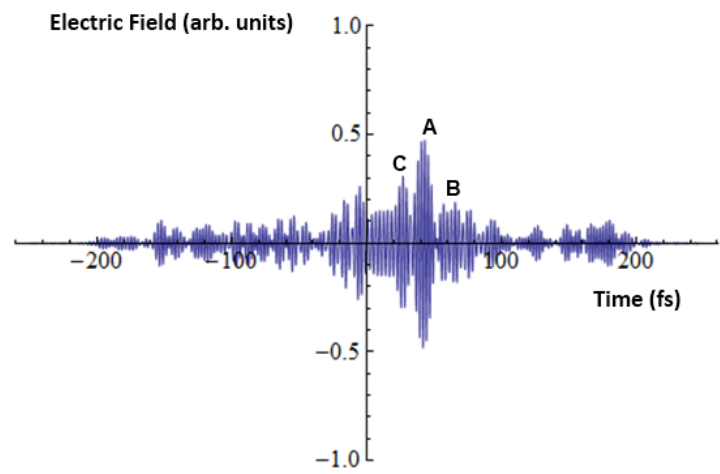

Figure 10: (Color online) Calculated pulse after passing through dye solution, with labeled reference peaks

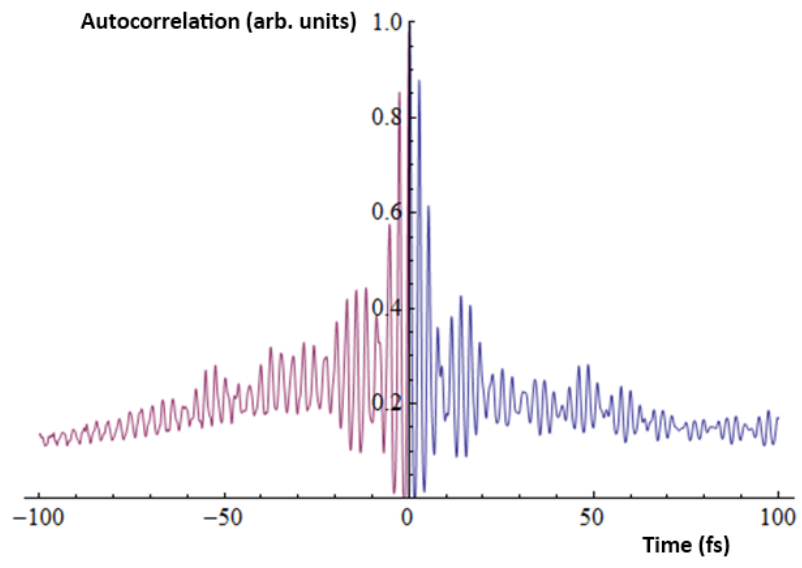

Figure 11: (Color online) Calculated autocorrelation from simulation (right half of graph, blue) and measured autocorrelation after propagation through dye solution (left half of graph, red)

be precisely calculated without knowledge of the thickness of the dye layer. However, the close match between the refractive indices of the BK7 glass $(\mathrm{n} \approx 1.51)$ and tetrachloroethane $(\mathrm{n} \approx 1.49)$ allows an Fresnel equation estimate of the reflection coefficient, yielding a reflectivity of less than $0.1 \%$. The additional effect of the dye dispersion on reflection can be estimated by setting a typical dye layer thickness of 2 microns in Eq. 4, which yields a negligible frequency-dependent change in the already small reflection coefficient. The intensity of multiply reflected pulses transmitted from within the dye layer is proportional to the square of the reflection coefficient, and can be neglected as well. After calculating the electric field of the propagated pulse at the experimentally accessible value of $\varphi=1$, we can compare the observed autocorrelation to the autocorrelation calculated from the simulated pulse. The calculated pulse after passing through the dye is presented in Figure 10, while both the calculated and observed autocorrelations are plotted in Figure 11.

Good agreement could be seen around the central portion, indicating the closest association between theory and experiment for the high-amplitude features in the calculated pulse. The theoretical autocorrelation is less accurate in the wings of the signal; this likely indicates that the genetically retrieved pulse may not well represent the smaller outer features of the pulse. We then calculate the pulse shape for dye propagation distances between 0 and the total dye thickness $\varphi=1$. We track the labeled features and measure their attenuation as the propagation distance is varied (Figure 12).

The pulse peak (feature A) decays exponentially with a best-fit dimensionless attenuation length of 0.528 while the secondary feature B decays with a much longer best-fit attenuation length of 0.297 and the secondary feature C decays with an intermediate attenuation length of 0.187 . This variation of attenuation length for each feature is a signature of precursor-like behavior. 


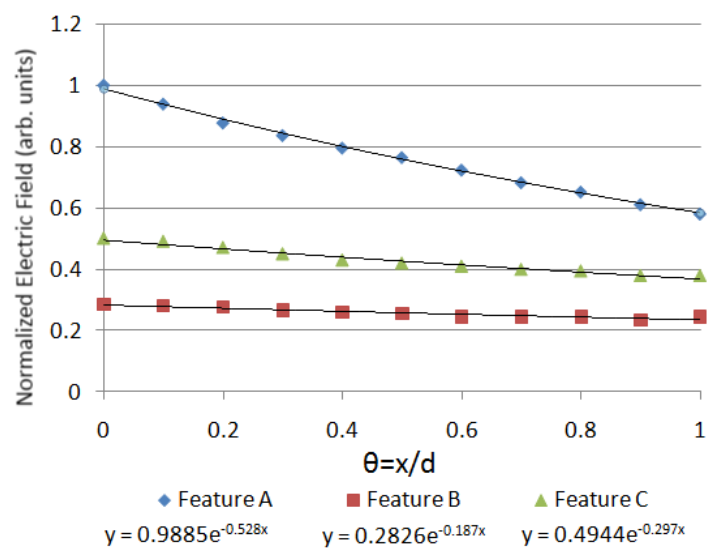

Figure 12: (Color online) Distance attenuation of selected time-domain pulse features with exponential best-fit curves

\section{SUMMARY}

We observe a signature for enhanced attenuation distance characteristic of optical precursors by means of intereferometric autocorrelation and genetic algorithm phase retrieval supplemented by numerical simulation. Limitations in the accuracy of the autocorrelation method prevent full knowledge of the retrieved electric field and precise characterization of precursor fields. However, the behavior of the of high-amplitude portion of the propagating field is readily discernible. Those features of the high-amplitude portion which correspond to few-cycle rise times exhibit reduced exponential attenuation with respect to distance. This behavior is consistent with current theoretical knowledge of precursor behavior.

\section{Acknowledgments}

The authors would like to acknowledge Will Buck for his help in the selection of the dye. This research was partially supported by the Office of Naval Research (ONR) under contract N00014-08-1-0037 and the Defense University Research Initiative Program (DURIP) under contract N00014-08-1-0804, the Robert A. Welch foundation (grants No. A-1546 and A-1547), and by the National Science Foundation (grants No. 072280 and 0555568).

[1] L. Brillouin, Wave Propagation and Group Velocity (Academic Press, Inc., 1960).

[2] J. Aaviksoo, J. Kuhl, and K. Ploog, Phys. Rev. A 44, 5353 (1991).

[3] H. Jeong, A. Dawes, and D. Gauthier, Phys. Rev. Lett. 96, 143901 (2006).

[4] D. Wei, J. Chen, M. Loy, G. Wong, and S. Du, Phys. Rev. L 103, 93602 (2009).

[5] S. Du, C. Belthangady, P. Kolchin, G. Yin, and S. Harris, Opt. Lett. 33, 2149 (2008).

[6] U. Österberg, D. Andersson, and M. Lisak, Opt. Comm. 277, 5 (2007).

[7] S. Choi and U. Österberg, Phys. Rev. Lett. 92, 193903 (2004).

[8] L. Naveira, B. Strycker, J. Wang, G. Ariunbold, A. Sokolov, and G. Kattawar, App. Opt. 48, 1828 (2009).

[9] T. Kohmoto, Y. Fukui, S. Furue, K. Nakayama, and Y. Fukuda, Phys. Rev. E. 74, 56603 (2006).

[10] J. Jackson, Classical Electrodynamics, 3rd ed (John Willey \& Sons, 1999).

[11] K. E. Oughstun and G. C. Sherman, Electromagnetic Pulse Propagation in Causal Dielectrics (Springer-Verlag, 1994).

[12] K. Oughstun, Proc. IEEE 79, 1379 (1991).

[13] J. Chung and A. Weiner, IEEE J. Quantum Electron. 7, 656 (2001).

[14] R. Trebino, Frequency-resolved Optical Gating: The Measurement of Ultrashort Laser Pulses (Kluwer, 2002).

[15] W. Yang, M. Springer, J. Strohaber, A. Kolomenski, H. Schuessler, G. Kattawar, and A. Sokolov, Optics Express 18, 15028 (2010). 